\title{
Plastic Flow in a Thin Layer: New Formulation of the Boundary- Value Problem and Its Solution
}

\author{
Vagid Kadymov, ${ }^{1, *}$ and Eugene Sosenushkin ${ }^{2}$ \\ ${ }^{1}$ Moscow State University of Humanities and Economics, RU-107150 Moscow, Russia \\ ${ }^{2}$ Moscow State Technological University "STANKIN", RU-127055, Moscow, Russia
}

\begin{abstract}
The paper is dedicated to the theory of flow in a thin plastic layer that has numerous applications in calculations of technological processes of material treatment by pressure, such as stamping and pressing of thin-walled structural elements, thin-sheet rolling, and others. Two-dimensional, averaged over the thickness of the layer mathematical formulation of the contact boundary value problem of the spreading of a plastic layer on the plane has been examined. In the framework of the general "viscous liquid" model, an approximate analytical solution has been obtained and evaluated against measurements. This solution is in good agreement with the experimental results in the area far from the free boundary and the central part of the sample.
\end{abstract}

\section{Introduction}

Problems of plastic flow of the material in a relatively thin layer between the converging surfaces of the tool bodies are important for solving many fundamental and practical problems [1-5] a fairly wide class of This includes most of the technological problems of material treatment by pressure, such as stamping and pressing of thin-walled structural elements, thin-sheet rolling and etc. In particular, there exist complex space problems of plastic shaping with a variety of parameters that determine the properties of the material being shaped. In these processes high pressures which are an order of magnitude higher than the shear properties of the layer material, develop, and, thus, the properties of the material in the layer can be approximated by those of a hydrodynamic liquid [1,5].

Almost along the entire contact surface, slippage is observed, in which the contact surfaces coincide with the sliding surfaces and the friction forces acting on them reach a maximum value equal to the yield strength of the layer material [3]. The accuracy requirements of the final part of processing define another important feature of these processes. It is wellknown[2] that high contact pressures cause normal elastic movements of the working surfaces of the tool bodies, commensurate with the thickness of the plastic layer, and, therefore, in this case, the neglection of the elastic movements of the tool bodies is unjustified. In high-speed processes of material treatment by pressure, the viscosity and inertia forces play an essential role that greatly complicates the modeling of the physical process where temperature and heat flows play an important role. Thus, heat generation due to the sliding friction forces on the contact surface and the dissipation of mechanical energy also must be considered. It is important to note that ihot processes of pressure treatment are associated with an intensive heat exchange with external bodies, which results in the hardening of the contact layers ("cold welding") at the initial stage of the process. In some cases, it is important to take into account the influence of pressure on the properties of the material being formed. The other important feature of such processes is that the boundary conditions in these processes are not defined and that the boundaries themselves are not known in advance.

Based on the analysis of the solution to L.Prandt problem, A.A. Ilyushin has formulated a number of hypotheses regarding the kinematics and the friction forces on the contact surface [1] . Then he utilized them to develop an effective two-dimensional theory of flow in a thin plastic layer that has become an important instrument of exploring these processes. Within the framework of this model, a general boundary value problem for a "viscous" liquid has been formulated. This problem is described by a set nonlinear partial differential equations of the second order with respect to three unknown functions: contact pressure and two components of the flow velocity $[1,2,5,10]$. It is important to note that until recently, all studies in this area were conducted on the basis of a simplified formulation of the boundary value problem involving a the model of an "ideal" fluid described by nonlinear partial differential equations of the first order, in which tangent stresses are neglected. In the framework of this simplified formulation, the kinematics of the spreading of the plastic layer was investigated and the evolutionary equation determining the free boundary of the spreading layer was obtained. In [5], a more sophisticated forms of the representation of the evolutionary equation have

e-mail: vkadymov@yandex.ru 
been introduced. Moreover, it has been found [5] that this equation can be reduced to a special case of the nonlinear equation of thermal conductivity.

This theory has been extended to the case of plastic flow on elastically deformable surfaces [2] and on surfaces with a pronounced texture implying anisotropy of the properties of friction forces on contact [6,7]. The theory has also been applied for the flows in high-speed processes [4] and in the treatment of volumetrically compressible material and material with structural dependence (powder, sintered material) [3].

However, not all flow processes in a thin plastic layer can be described in the framework of a simplified model of an "ideal" liquid. Recently, such cases have been studied theoretically (the spreading of a plastic layer consisting of two bordering different media [8]), and experimentally (the spreading of the layer in the area with a fixed boundary [9, 11]). In such cases, for a correct description of these processes, it is necessary to use a general model of "viscous" liquid, which we will be explored and discussed here.

\section{Mathematical formulation of the boundary-value problem of the spreading of a plastic layer}

The basic equations of the boundary-value problem of plastic layer flow on the plane have the form $[1,4,5]$ :

$$
\begin{gathered}
\frac{\partial p}{\partial x}=\frac{\sigma_{s}}{3} \frac{d t}{d \lambda} \Delta u-\frac{2 \tau_{s}}{h} \frac{u}{\sqrt{u^{2}+v^{2}}}, \\
\frac{\partial p}{\partial y}=\frac{\sigma_{s}}{3} \frac{d t}{d \lambda} \Delta v-\frac{2 \tau_{s}}{h} \frac{v}{\sqrt{u^{2}+v^{2}}}, \\
\frac{\partial u}{\partial x}+\frac{\partial v}{\partial y}-\frac{d \lambda}{d t}=0
\end{gathered}
$$

where $p, u, v$ are the contact pressure and components of flow velocity; $\sigma_{s}=\sqrt{3} \tau_{s}$ is the yield strength of plastic material; $h=h(t)$ is the known law of change of the layer thickness; $\lambda(t)=\ln \left(h\left(t_{0}\right) / h(t)\right)$ is the degree of deformation, where $t_{0}$ is some initial point of time.

On a free previously unknown boundary the two scalar dynamic conditions are specified:

$$
\begin{aligned}
& \vec{\sigma}^{(n)}=\tilde{\sigma} \cdot \vec{n}= \\
& \left(\sigma_{x x} n_{x}+\sigma_{x y} n_{y} ; \sigma_{x y} n_{x}+\sigma_{y y} n_{y}\right)=\overrightarrow{0},
\end{aligned}
$$

and the kinematic condition is given:

$$
v_{n} \equiv \vec{v} \cdot \vec{n}=-\frac{\partial F / \partial t}{|\operatorname{gradF}|},
$$

At the tool contact boundary (usually known) $\Gamma_{\sigma}: F(x, y, t)=0$, the following two conditions are set: one dynamic condition with respect to the shear stress

$$
\begin{aligned}
& \left|\vec{\sigma}^{(n)} \cdot \vec{\tau}\right|=\left|\sigma_{x x} n_{x} n_{y}+\sigma_{x y}\left(n_{y}^{2}-n_{x}^{2}\right)-\sigma_{y y} n_{x} n_{y}\right|= \\
& =\mu \tau_{s}, \quad(0 \leq \mu \leq 1),(6)
\end{aligned}
$$

and one kinematic condition of the impermeability

$$
v_{n} \equiv \vec{v} \cdot \vec{n}=-\frac{\partial G / \partial t}{|\operatorname{grad} G|},
$$

where $\vec{\tau}$ and $\vec{n}$ are the unitary tangent and normal vectors with respect to $\Gamma_{c}$. In particular, at the fixed boundary, the normal velocity is zero.

Let's move on to dimensionless variables. Let $\sigma_{s}, L_{0}, h_{0},\left(d h\left(t_{0}\right) / d t\right)$ be the characteristic values of the yield strength of the material, the linear size and thickness of the layer, and the rate of convergence of the plates. We introduce a small parameter $\varepsilon=h_{0} / L_{0}<<1$ , characterizing the fineness of the plastic layer. The analysis of incompressibility equation leads to: a) $t_{0}=L_{0} / v_{0}$;b) $v_{0}=-\left(d h\left(t_{0}\right) / d t\right) / \varepsilon$. The latter means that the characteristic value of the flow velocity along the layer is much greater than the rate of convergence of the plates, which is commonly expected. Let's consider $p_{0}$ as the characteristic value of the contact pressure in the plastic layer. The analysis of the quasistatic equilibrium equations shows that the characteristic value of the contact pressure is much higher than the yield strength of the layer material, i.e. $p_{0}=\sigma_{s} / \varepsilon$. We now have the following system of equations in dimensionless form:

$$
\begin{gathered}
\frac{\partial \bar{p}}{\partial \bar{x}}=\varepsilon \frac{d \bar{t}}{d \lambda} \bar{\Delta} \bar{u}-\frac{2}{\sqrt{3}} \frac{1}{\bar{h}} \frac{\bar{u}}{\sqrt{\bar{u}^{2}+\bar{v}^{2}}}, \\
\frac{\partial \bar{p}}{\partial \bar{y}}=\varepsilon \frac{d \bar{t}}{d \lambda} \bar{\Delta} \bar{v}-\frac{2}{\sqrt{3}} \frac{1}{\bar{h}} \frac{\bar{v}}{\sqrt{\bar{u}^{2}+\bar{v}^{2}}}, \\
\frac{\partial \bar{u}}{\partial \bar{x}}+\frac{\partial \bar{v}}{\partial \bar{y}}-\frac{d \lambda}{d \bar{t}}=0,
\end{gathered}
$$

where dimensionless quantities are marked by a dash at the top, $\bar{h}=h / h\left(t_{0}\right)$. 
The conditions at the free boundary $\Gamma_{\sigma}: \bar{F}(\bar{x}, \bar{y}, \bar{t})=0$ :

$$
\begin{gathered}
{\left[-\bar{p}+\left(\frac{2}{3}+\frac{2}{3} \frac{d \bar{t}}{d \lambda} \frac{\partial \bar{u}}{\partial \bar{x}}\right) \varepsilon\right] n_{x}+} \\
+\left[\frac{1}{3} \frac{d \bar{t}}{d \lambda}\left(\frac{\partial \bar{u}}{\partial \bar{y}}+\frac{\partial \bar{v}}{\partial \bar{x}}\right) \varepsilon\right] n_{y}=0, \\
+\left[\frac{1}{3} \frac{d \bar{t}}{d \lambda}\left(\frac{\partial \bar{u}}{\partial \bar{y}}+\frac{\partial \bar{v}}{\partial \bar{x}}\right) \varepsilon\right] n_{x}+ \\
\left.+\bar{p}+\left(\frac{2}{3}+\frac{2}{3} \frac{d \bar{t}}{d \lambda} \frac{\partial \bar{v}}{\partial \bar{y}}\right) \varepsilon\right] n_{y}=0, \\
\bar{v}_{n}=-\frac{\partial \bar{F} / \partial \bar{t}}{|\operatorname{grad} \bar{F}|}
\end{gathered}
$$

The conditions at the tool contact boundary $\Gamma_{c}$ :

$$
\begin{gathered}
{\left[-\bar{p}+\left(\frac{2}{3}+\frac{2}{3} \frac{d \bar{t}}{d \lambda} \frac{\partial \bar{u}}{\partial \bar{x}}\right) \varepsilon\right] n_{x} n_{y}+} \\
+\left[\frac{1}{3} \frac{d \bar{t}}{d \lambda}\left(\frac{\partial \bar{u}}{\partial \bar{y}}+\frac{\partial \bar{v}}{\partial \bar{x}}\right) \varepsilon\right]\left(n_{y}^{2}-n_{x}^{2}\right)- \\
-\left[-\bar{p}+\left(\frac{2}{3}+\frac{2}{3} \frac{d \bar{t}}{d \lambda} \frac{\partial \bar{v}}{\partial \bar{y}}\right) \varepsilon\right] n_{x} n_{y}= \pm \frac{\mu}{\sqrt{3}} \varepsilon \\
\bar{v}_{n}=-\frac{\partial \bar{G} / \partial \bar{t}}{|\operatorname{grad} \bar{G}|}
\end{gathered}
$$

The system of differential equations (1)'-(3)' together with the boundary conditions (4)' - (7)' constitutes the general boundary-value problem of the spreading of a "viscous fluid".

In the main approximation $(\varepsilon=0)$, the original equations are simplified by decreasing the order of the system:

$$
\begin{aligned}
& \frac{\partial \bar{p}}{\partial \bar{x}}=-\frac{2}{\sqrt{3}} \frac{1}{\bar{h}} \frac{\bar{u}}{\sqrt{\bar{u}^{2}+\bar{v}^{2}}}, \\
& \frac{\partial \bar{p}}{\partial y}=-\frac{2}{\sqrt{3}} \frac{1}{\bar{h}} \frac{\bar{v}}{\sqrt{\bar{u}^{2}+\bar{v}^{2}}}, \\
& \frac{\partial \bar{u}}{\partial \bar{x}}+\frac{\partial \bar{v}}{\partial \bar{y}}-\frac{d \lambda}{d \bar{t}}=0
\end{aligned}
$$

It is clear that the number of boundary conditions for the simplified problem of the "ideal fluid" also decreases.

\section{Results and Discussion}

In this part we introduce a new formulation of the boundary value problem of the plastic layer spreading. Based on this new formulation, we will obtain a solution of the problem of the plastic layer spreading in the rectangular area at its initial state.

We introduce the current function $\psi=\psi(x, y)$ so that the incompressibility condition (2) is satisfied:

$$
u=\frac{d \lambda}{d t}\left(\psi_{y}^{\prime}+\frac{x}{2}\right), \quad v=-\frac{d \lambda}{d t}\left(\psi_{x}^{\prime}-\frac{y}{2}\right)
$$

Substitute (9) in the quasistatic equilibrium equations, as a result there is a system of two differential equations with respect to $p$ and $\psi$ :

$$
\frac{p_{x}^{\prime}-\left(\sigma_{s} / 3\right) \Delta \psi_{y}^{\prime}}{p_{y}^{\prime}+\left(\sigma_{s} / 3\right) \Delta \psi_{x}^{\prime}}=-\frac{2 \tau_{s}}{h} \frac{\psi_{y}^{\prime}+x / 2}{\psi_{x}^{\prime}-y / 2},
$$

$$
\begin{gathered}
\left(p_{x}^{\prime}-\left(\sigma_{s} / 3\right) \Delta \psi_{y}^{\prime}\right)^{2}+ \\
+\left(p_{y}^{\prime}+\left(\sigma_{s} / 3\right) \Delta \psi^{\prime}\right)^{2}=\frac{4 \sigma_{s}^{2}}{3 h^{2}},
\end{gathered}
$$

Below we examine in dimensional quantities the problem of the spreading of the plastic layer in the area with the shape of a rectangle (Fig.1) at the initial state in a plan.

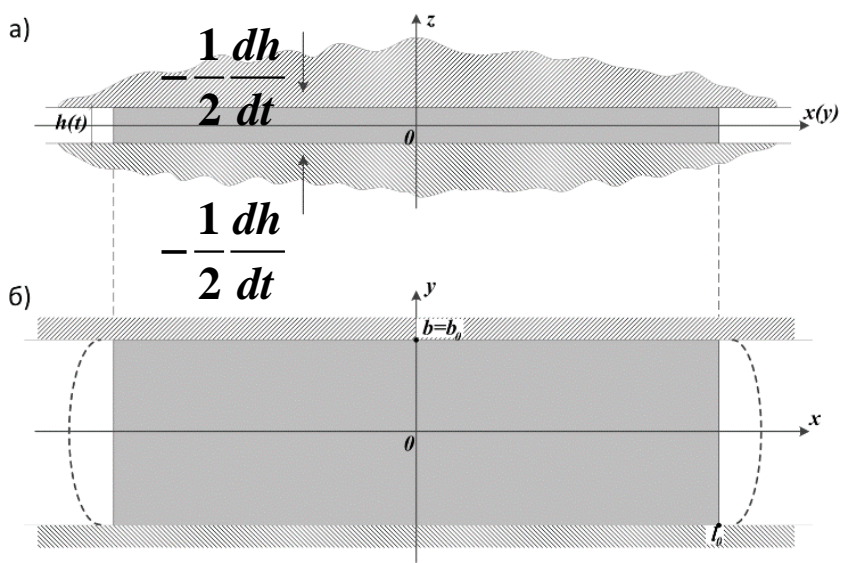

Fig.1. Spreading of the plastic layer in the cross section (a), and in the plan (b).

Using the symmetry of the domain, we consider the problem in the first quadrant of the plane. On a fixed wall $\left(G(x, y) \equiv y-b_{0}=0\right)$ :

$$
v=0 \Rightarrow \psi_{x}^{\prime}-\frac{b_{0}}{2}=0
$$

$$
\frac{\partial u}{\partial y}+\frac{\partial v}{\partial x}=-\sqrt{3} \lambda^{\prime} \mu \Rightarrow \psi_{y y}^{\prime \prime}-\psi_{x x}^{\prime \prime}=-\sqrt{3} \lambda^{\prime} \mu
$$


The boundary conditions on the symmetry lines of the flow area take the following form:

$$
\begin{aligned}
& y=0: \quad v=0 \Rightarrow \psi_{x}^{\prime}=0, \quad \frac{\partial u}{\partial y}=0 \Rightarrow \psi_{y y}^{\prime \prime}=0 \\
& x=0: \quad u=0 \Rightarrow \psi_{y}^{\prime}=0, \quad \frac{\partial v}{\partial x}=0 \Rightarrow \psi_{x x}^{\prime \prime}=0
\end{aligned}
$$

At the unknown free boundary $F(x, y, t)=0$, which at the initial point of time has the form $x=l_{0}\left(n_{x}=1, n_{y}=0\right)$, the boundary conditions are simplified:

$$
\begin{gathered}
\sigma_{x x}=0 \Rightarrow-p+\sigma_{s}+\frac{2}{3} \sigma_{s} \psi_{x y}^{\prime \prime}=0, \\
\sigma_{x y}=0 \Rightarrow \psi_{y y}^{\prime \prime}-\psi_{x x}^{\prime \prime}=0 .
\end{gathered}
$$

Let's try to find a solution that is far away from the line $x=0$. To do this, we assume that at the considered area of flow $v(x, y)=0$.

As a result of the integration of equation (3) we have:

$$
u(x, y)=\lambda^{\prime}[x+g(y)]
$$

where the function $g(y)$ must be determined from the other equations. From equation (2) we obtain that $p=p(x)$. As a result, equation (1) takes the form:

$$
p^{\prime}(x)=\frac{\sigma_{s}}{3} g^{\prime \prime}(y)-\frac{2}{\sqrt{3}} \frac{\sigma_{s}}{h},
$$

where the left part represents a function of the variable $x$, and the right part depends on $y$ (and parameter $h(t))$. Therefore, the variables are separated, and we have the system with an arbitrary constant (function of time) $k$ :

$$
g^{\prime \prime}(y)=k=\text { const, } \quad p^{\prime}(x)=\frac{\sigma_{s} k}{3}-\frac{2 \sigma_{s}}{\sqrt{3} h} \text {. }
$$

We integrate the first of these equations (18) taking into account the boundary conditions (13), (14):

$$
\begin{gathered}
g(y)=c-\frac{\sqrt{3} \mu}{2 b_{0}} y^{2}, \quad k=-\frac{\sqrt{3} \mu}{b_{0}}, \\
u(x, y)=\lambda^{\prime}\left[x-\frac{\sqrt{3} \mu}{2 b_{0}} y^{2}+c\right] .
\end{gathered}
$$

where the integration constant $C$ is still undefined.

It is worth noting that the first term in the right part (17) ' corresponds to the flow velocity in the model of "ideal fluid", in which the shear stress is zero.
Let's assume that the line $x=x_{f}(t)$ is a conditional free bound $\left(x_{f} h=x_{0} h_{0}\right)$.We then use the integral condition of the incompressible material volume conservation

$$
-x_{f}(t) \frac{d h}{d t} b_{0}=\int_{0}^{b_{0}} h u\left(x_{f}, y\right) d y,
$$

from which we determine the constant $c=\frac{\sqrt{3}}{6} \mu b_{0}$, included in the solution. As a result, the flow velocity takes the form:

$$
u(x, y)=\lambda^{\prime}\left[x+\frac{\sqrt{3}}{6 b_{0}}\left(\mu b_{0}^{2}-3 y^{2}\right)\right]
$$

It is now evident that the boundary condition (15) is satisfied in the integral form:

$$
\int_{0}^{b_{0}} u(0, y) d y=\frac{\lambda^{\prime} \sqrt{3}}{6 b_{0}} \int_{0}^{b_{0}}\left(b_{0}^{2}-3 y^{2}\right) d y=0 .
$$

Integration of the second equation of the system (18) gives:

$$
p(x)=p_{0}-\frac{\sigma_{s} x}{\sqrt{3}}\left(\frac{2}{h}+\frac{\mu}{b_{0}}\right),
$$

with integration constant $p_{0}$. It can be seen that the pressure does not depend on $y$.

Assuming that at the free boundary the normal stress is zero:

$$
\left(\sigma_{x x}=\right)-p+4 \sigma_{s} / 3=0 \text {, }
$$

according to (20) we find:

$$
p_{0}(t)=\frac{2 \sigma_{s} x_{f}}{\sqrt{3} h}+\left(\frac{x_{f} \mu}{\sqrt{3} b_{0}}+\frac{4}{3}\right) \sigma_{s} .
$$

With a sufficiently large $x_{f}$, the last (third) term in the right part can be neglected, then

$$
p_{0}(t)=\frac{\sigma_{s} x_{f}}{\sqrt{3}}\left(\frac{2}{h}+\frac{\mu}{b_{0}}\right),
$$

and the pressure in (20) has a simpler form:

$$
p \approx \frac{\sigma_{s}}{\sqrt{3}}\left(\frac{2}{h}+\frac{\mu}{b_{0}}\right)\left(x_{f}-x\right) .
$$

At the end of the work we write down the function of the current $\psi=\psi(x, y)$ : 


$$
\begin{aligned}
& \psi_{u}^{\prime}=\frac{y}{2} \\
& \psi_{y}^{\prime}=\frac{x}{2}+\frac{\sqrt{3}}{6 b_{0}}\left(\mu b_{0}^{2}-3 y^{2}\right) \Rightarrow \\
& \psi(x, y)=\frac{\sqrt{3}}{6 b_{0}}\left(\mu b_{0}^{2} y-y^{3}\right)+c_{1}
\end{aligned}
$$

Thus, an approximate analytical solution is obtained within the framework of the general model of the "viscous liquid". It is worth noting that the obtained solution satisfies the boundary conditions in the integral form both at the free boundary $x=x_{f}(t)$ and at the boundary $x=0$. Therefore, according to the principle of Saint-Venant, the solution is valid in the central part of the flow area.

We can observe the inhomogeneous nature of the flow, in which initially straight lines ( $X=$ const) are curved over time, and there is a significant lag in the longitudinal movement of material next to the fixed boundary. This fact is confirmed by the results of the experiments $[4,5]$. The obtained regularities cannot be described with the model of the "ideal fluid", in which tangential stresses are neglected.

\section{Conclusion}

The paper focuses on the theory of flow in a thin plastic layer, which was originally developed by A.A. Ilyushin and has served as a guide in calculations of technological processes of material treatment by pressure, such as stamping and pressing of thin-walled structural elements, thin-sheet rolling, and others, for many years This paper discusses the current developments of mathematical modeling of contact problems of plastic flow in a thin layer and present an approximate analytical solution, obtained within the framework of the general "viscous liquid" model, that has been corroborated by comparing modeling results with is experimental data [8,9]. The new dependencies obtained in this study cannot be described using "ideal fluid" model, in which tangential stresses are neglected.
Numerous important issues yet to be resolved include consideration of the elastic deformation of the tool, the volumetric compressibility of the processed material, the use and retention of intermediate soft media along the contact surface throughout the process, the use and implementation of tools with relief-expressed contact surfaces, modeling of complex loading and impacts of super plasticity in technological processes, and others. Further research, both theoretical and experimental, is needed in order to resolve these issues.

This work was carried out using equipment provided by the Center of Collective Use of MSUT "STANKIN".

\section{References}

1. A.A. Ilyushin, Proceedings (1946-1966) Vol.2 Plasticity, (M.:Fizmatlit. 2004). ISBN 5-9221-03644

2. I.A. Kiyko, Theory of plastic flow (M.: MSU. 1978).

3. I.A. Kiyko, Bulletin of Moscow University. S.1, 4, 47-52 (2002)

4. I.A. Kiyko, V.A. Kadymov, Bulletin of Moscow University. S.1, 4, 50-56 (2003)

5. N. Belov, V. Kadymov, Mechanics of solids, 46, 3646 (2011)

6. V. Kadymov, Nonlinear Analysis: Theory \& Appl., 30(8), 5259-5265 (1997)

7. I.A. Kiyko, Applied mathematics and mechanics, 70, 344-351 (2006)

8. V.A. Kadymov, Proceedings of Moscow University MAMI, 2(27), 323-330 (2012)

9. N.A. Belov, V.A. Kadymov, E.N. Sosenushkin, Prepr. №1100. Institute of Problems of Mechanics. RAS, 23 (2015)

10. V. Kadymov, Mathematical Methods in the Applied Sciences, 38(16), 3421-3431 (2015)

11. V.A. Kadymov, Mathematical modeling of contact problems of plastic flow, (Monograph. Germany: Palmarium Academic Publishing, 2016). 